\title{
Group Scheduling with General Autonomous and Induced Learning Effect
}

\author{
Jia-zhen Huo, ${ }^{1}$ Lei Ning $\left(\mathbb{D},{ }^{1}\right.$ and Li Sun ${ }^{2}$ \\ ${ }^{1}$ School of Economics and Management, Tongji University, Shanghai 200070, China \\ ${ }^{2}$ School of Business, Shanghai Dianji University, Shanghai 201306, China \\ Correspondence should be addressed to Lei Ning; ninglei1985@126.com
}

Received 6 April 2018; Revised 19 June 2018; Accepted 10 July 2018; Published 5 August 2018

Academic Editor: Łukasz Jankowski

Copyright (c) 2018 Jia-zhen Huo et al. This is an open access article distributed under the Creative Commons Attribution License, which permits unrestricted use, distribution, and reproduction in any medium, provided the original work is properly cited.

\begin{abstract}
In recent research, induced learning requires management involvement. The initial investment can be interpreted as induced learning via an investment in the creation of technological knowledge or knowledge transfer. Induced learning has received attention in productivity improvement. In this paper, we consider scheduling problems with autonomous and induced learning under group technology. The jobs' processing times decrease according to a learning rate which conversely increases the investment cost. The objectives of the scheduling problems are to minimize a linear combination of the makespan and the investment cost and a linear combination of the total completion time and the investment cost. We present polynomial algorithms to determine the optimal job sequence, group sequence, and the percentage reduction of the standard learning rate.
\end{abstract}

\section{Introduction}

Jobs' actual processing times usually decrease as a result of workers' improved skill due to repetitive performance of similar jobs. This process is known as the learning effect in the literature. Biskup [1], Wright [2], and Cheng and Wang [3] are among the pioneers that brought the topic of learning into the field of scheduling. Recent studies that consider scheduling with the learning effect include Qian and Steiner [4], Wang and Wang [5], Rudek [6], Liu [7], Shen and Wu [8], Wu et al. [9], Li et al. [10], and Rudek [11]. In the recent literature, a quality improvement of labour due to repetition is the factor in productivity increasing, which is called autonomous learning or learning by doing. As opposed to autonomous learning, which is a by-product of repeating activities, induced learning requires management involvement. The initial investment can be interpreted as induced learning via an investment in the creation of technological knowledge or knowledge transfer. Induced learning has received attention in productivity manufacturing. Nevertheless, it is hardly considered in scheduling. Most existing scheduling models with the learning effect concentrate on autonomous learning, i.e., learning by doing. Zhang et al. [12] considered scheduling problems with autonomous and induced learning. Biskup and Simons [13] investigated scheduling problems with autonomous and induced learning. In addition to these two papers, there is no research, which considers scheduling problems with autonomous and induced learning simultaneously.

In many manufacturing processes, production efficiency can be raised by grouping various parts and products with similar designs or requiring similar production processes. This practice is known as group technology in the literature. Many advantages of applying group technology in manufacturing have been observed. Research considering group technology includes Wei and Wang [14], Yang [15], Lee and Wu [16], Bai et al. [17], and Lee and Lu [18].

To the best of our knowledge, scheduling with autonomous and induced learning under group technology has not been studied in the literature. The topic pertains to the scope of mathematical problems in engineering. Therefore, we think this paper will arouse reader's interest. In this paper, we introduce group scheduling problems with autonomous and induced learning. We present that the problems to minimize the weighted sum of the makespan and the investment cost and the weighted sum of the total 
completion time and the investment cost are polynomially solvable. The remaining part of this paper is organized as follows. In Section 2, we formulate the problem and introduce the notation and some lemmas. We then study the problem to minimize the weighted sum of the makespan and the investment cost. In Section 3, we consider the problem to minimize the weighted sum of the total completion time and the investment cost. In the last section, we conclude the paper.

\section{Problem Formulation}

There are $n$ jobs to be classified into $m$ groups, where the number of jobs within each group is $n_{i}, i=1,2, \ldots, m$. That is to say, a job belonging to a group is decided in advance. All the jobs are ready to be processed on a single machine and are nonpreemptive. Jobs in the same group are processed consecutively and need no setup time. A group setup time is required if the machine switches processing jobs from one group to another. We first define the notation which are used throughout this paper and formulate the problems under study.

$G_{[i]}$ : group scheduled in the $i$ th position in a sequence, $i=1,2, \ldots, m$.

$n_{i}$ : the number of jobs in group $G_{i}, i=1,2, \ldots, m$.

$n$ : the total number of jobs, i.e., $n_{1}+n_{2}+n_{3}+\cdots+n_{m}=n$. $s_{i}$ : the normal setup time of group $G_{i}, i=1,2, \ldots, m$. $s_{i[r]}^{A}$ : the actual setup time of group $G_{i}$ occupying the $r$ th group position in the sequence, $i=1,2, \ldots, m$.

$s_{[i]}^{A}$ : the actual setup time of group $G_{[i]}$ occupying the $i$ th group position in the sequence, $i=1,2, \ldots, m$.

$J_{i[j]}$ : the job scheduled in the $j$ th position in group $G_{i}$. $p_{i j}$ : the basic (normal) processing time of job $J_{i j}, i=$ $1,2, \ldots, m, j=1,2,3, \ldots, n_{i}$.

$p_{i[j]}$ : the basic (normal) processing time of job $J_{i[j]}$ scheduled in the $j$ th position of group $G_{i}, i=$ $1,2, \ldots, m, j=1,2,3, \ldots, n_{i}$.

$p_{i j[r, k]}^{A}$ : the actual processing time of job $J_{i j}$ scheduled in the $r$ th position of group position and the $k$ th jobposition in group $G_{i}, i=1,2, \ldots, m, j=1,2,3, \ldots, n_{i}$.

In this paper, we study the group scheduling problems under autonomous and induced learning. Following Biskup and Simins (2004), we assume that the actual setup time of group $G_{i}$ is scheduled in the $r$ th position as follows:

$$
s_{i[r]}^{A}=s_{i} r^{a \log _{2}(1-x) s}, \quad i=1,2, \ldots, m .
$$

$S$ is a learning rate; its value needs to be smaller than or equal to one as Biskup and Simins (2004). We consider job processing time model concerning autonomous and induced learning. Following the model in Lee and Wu [19], the actual processing time of job $J_{i j}$ when scheduled in the $r$ th group and in the $k$ th position is

$$
\begin{aligned}
p_{i j[r, k]}^{A}=p_{i j} r^{a \log _{2}(1-x) s} k^{b \log _{2}(1-x) s}, \\
\quad i=1,2, \ldots, m, j=1,2,3, \ldots, n_{i},
\end{aligned}
$$

where $x, 0 \leq x \leq x_{\max }<1$, is the percentage reduction of the standard learning rate $s$ and $0<s \leq 1$. There is an additional cost $k(x)$ incurred by the effort to achieve $x$, i.e., the investment cost. So the problem is to make a tradeoff between the original objective function and the investment cost through a proper choice of $x . a>0, b>0$ are the learning coefficient, which are related to jobs repetition and groups repetition. When $a=b=1$, it is the model studied in Biskup and Simous (2004). The objectives are to find the optimal job sequence in each group, the optimal group sequence, and the optimal investment cost such that the weighted sum of the makespan and the investment cost is minimized. Let AILE denote the jobs' autonomous and induced learning. Using the three-field notation for scheduling problems, we denote our problems as

$$
1\left|G T, s_{i[r]}^{A}=s_{i} r^{a \log _{2}(1-x) s}, A I L E\right| \beta_{1} C_{\max }+\beta_{2} k(x) .
$$

We also investigate the problem to minimize the weighted sum of the total completion time and the investment cost under the condition that all the groups have the same number of jobs, i.e.,

$$
\begin{aligned}
& 1\left|G T, s_{i[r]}^{A}=s_{i} r^{a \log _{2}(1-x) s}, A I L E, n_{i}=\bar{n}\right| \beta_{1} \sum C_{i j} \\
& \quad+\beta_{2} k(x) .
\end{aligned}
$$

where $\beta_{1}$ and $\beta_{2}$ are positive parameters decided by the decision-maker reflecting the relative weights of the two cost components. Before presenting the main results, we first present several lemmas that will be used in the proofs in the sequel.

Lemma 1. If $G(r)=\delta(r) r^{m \log _{2}(1-x) s}, m \geq 1,0 \leq x \leq x_{\text {max }}<$ $1, r \geq 1, \delta(r) \geq 1$, and $\delta(r)$ is a nonincreasing function, then $G(r)$ is nonincreasing function in $r$.

Lemma 2. If $h(x)=\delta(r) r^{m \log _{2}(1-x) s}, m \geq 1, r \geq 1,0 \leq x \leq$ $x_{\max }<1, \delta(r) \geq 1$, and $\delta(r)$ is a nonincreasing function, then $h(x)$ is a convex function over the interval $\left[0, x_{\text {max }}\right]$.

Lemma 3. If $\rho(x)=\delta_{1}(i, j) i^{a \log _{2}(1-x) s}+$ $\delta_{2}(i, j) i^{a \log _{2}(1-x) s} j^{b \log _{2}(1-x) s}, a \geq 1, b \geq 1,0 \leq x \leq x_{\text {max }}<1$, $i \geq 1, j \geq 1, \delta_{1}(i, j) \geq 1, \delta_{2}(i, j) \geq 1$, and $\delta_{1}(i, j)$ and $\delta_{2}(i, j)$ are nonincreasing functions with respect to $i$ and $j$, then $\rho(x)$ is a convex function over the interval $\left[0, x_{\max }\right]$.

Lemma 4 (Hardy et al. [20]). Let there be two sequences of numbers $x_{i}$ and $y_{i}$. The sum $\sum_{i} x_{i} y_{i}$ of products of the corresponding elements is the least (largest) if the sequences are monotonic in the opposite (same) sense. 


\section{The Makespan with the Investment Cost Minimization Problem}

In this section we consider the problem $1 \mid G T, s_{i[r]}^{A}=$ $s_{i} r^{a \log _{2}(1-x) s}, A I L E \mid \beta_{1} C_{\text {max }}+\beta_{2} k(x)$. Our objective is to find the optimal group sequence, the job sequence, and the percentage reduction of the standard learning rate $x$.

For the problem $1\left|G T, s_{i[r]}^{A}=s_{i} r^{a \log _{2}(1-x) s}, A I L E\right| \beta_{1} C_{\text {max }}+$ $\beta_{2} k(x)$, by calculation, we obtain the following expression:

$$
\begin{aligned}
& \beta_{1} C_{\max }+\beta_{2} k(x) \\
& =\sum_{i=1}^{m} \beta_{1} s_{[i]} i^{a \log _{2}(1-x) s} \\
& \quad+\sum_{i=1}^{m} \sum_{j=1}^{n_{i}} \beta_{1} p_{[i][j]} i^{a \log _{2}(1-x) s} j \log ^{b(1-x) s}+\beta_{2} k(x) .
\end{aligned}
$$

Let $f_{1}(x)=\sum_{i=1}^{m} \beta_{1} s_{[i]} i^{a \log _{2}(1-x) s}+$ $\sum_{i=1}^{m} \sum_{j=1}^{n_{i}} \beta_{1} p_{[i][j]} i^{a \log _{2}(1-x) s} j^{b \log _{2}(1-x) s}$ and $F_{1}(x)=$ $f_{1}(x)+\beta_{2} k(x)$. From Lemma 3 , we obtain that $f_{1}(x)$ is a convex function over the interval $\left[0, x_{\text {max }}\right]$. Since $k(x)$ is a convex function, $F_{1}(x)$ is also a convex function over the interval $\left[0, x_{\max }\right]$. Hence, for a certain $x \in\left[0, x_{\max }\right]$, the optimal sequence does not change (see Biskup and Simons [13]). Based on the above expression, for a certain $x \in\left[0, x_{\max }\right]$, the groups sequence does not affect the jobs sequence in each group.

Let $G_{1}(j)=\beta_{1} j^{b \log _{2}(1-x) s}$. From Lemma 1 , we see that $G_{1}(j)$ is a nonincreasing function about $j$. From Lemma 4 , for a given group sequence, the optimal internal job sequence can be obtained by assigning $p_{i[j]}$ to the corresponding $G_{1}(j)$ following a well-known result on two vectors given by Hardy et al. [20]. Since $G_{1}(j)$ is a nonincreasing function about $j, p_{i[j]}$ should be a nondecreasing sequence. Therefore, the optimal schedule is obtained by sequencing the jobs in each group in nondecreasing order of $p_{i[j]}$, i.e., $p_{i[1]} \leq p_{i[2]} \leq$ $, \ldots, \leq p_{i\left[n_{i}\right]}, i=1,2,3, \ldots, m$ (the shortest processing time $\mathrm{SPT}$ rule). For a certain $x \in\left[0, x_{\max }\right]$, if the internal job sequence in each group is determined, the total cost depends on the group sequence. Consequently, we show that the group sequence is determined by the following assignment problem:

$$
\begin{aligned}
\text { Minimize } & Z=\sum_{i=1}^{m} \sum_{r=1}^{m} w_{i r} x_{i r} \\
\text { s.t. } & \sum_{i=1}^{m} x_{i r}=1 \quad r=1,2, \ldots, m, \\
& \sum_{r=1}^{m} x_{i r}=1 \quad i=1,2, \ldots, m, \\
& x_{i r}=0 \text { or } 1 \\
& i=1,2, \ldots, m, r=1,2, \ldots, m .
\end{aligned}
$$

where $w_{i r}=\beta_{1} s_{i} r^{a \log _{2}(1-x) s}+\sum_{j=1}^{n_{i}} \beta_{1} p_{i[j]} r^{a \log _{2}(1-x) s} j^{b \log _{2}(1-x) s}$.
From the above formulation, the optimal $x$ depends on the sign of the first derivative of $F_{1}(x)$, i.e., $F_{1}^{\prime}(x)$. If $F_{1}^{\prime}(x)$ is positive, we should allocate the minimal value to $x$, i.e., $x=0$. If $F_{1}^{\prime}(x)$ is negative, we should allocate the maximal value to $x$, i.e., $x=x_{\text {max }}$. If $F_{1}^{\prime}(x)$ is equal to 0 , any value between 0 and $x_{\text {max }}$ will not affect the total cost.

Based on the above analysis, we present the following algorithm to solve this problem.

\section{Algorithm 5.}

Step 1. Arrange the jobs in each group in the SPT order, i.e.,

$$
p_{i[1]} \leq p_{i[2]} \leq, \ldots, \leq p_{i\left[n_{i}\right]}, \quad i=1,2, \ldots, m .
$$

Step 2. Give a value to $x$, which $x \in\left[0, x_{\max }\right]$, schedule the groups by $\left(A P_{1}\right)$.

Step 3. Take the first derivative of $F_{1}(x)$

Set

$$
x^{*}= \begin{cases}0, & \text { if } F_{1}^{\prime}(x)>0, \\ x_{\text {max }}, & \text { if } F_{1}^{\prime}(x)<0, \\ x \in\left[0, x_{\text {max }}\right], & \text { if } F_{1}^{\prime}(x)=0 .\end{cases}
$$

Step 4. Output the optimal job sequence, optimal group sequence, and optimal percentage reduction of the standard learning rate $x^{*}$.

Step 1 requires $O\left(\sum_{i=1}^{m} n_{i} \log n_{i}\right)=O(n \log n)$ time. Step 2 is executed in $O\left(\mathrm{~m}^{3}\right)$. Therefore, the complexity of Algorithm 5 is at most $O\left(n^{3}\right)$. Thus, we have obtained the following result.

Theorem 6. The $1\left|G T, s_{i[r]}^{A}=s_{i} r^{a \log _{2}(1-x) s}, A I L E\right| \beta_{1} C_{\text {max }}+$ $\beta_{2} k(x)$ problem is solvable in $\mathrm{O}\left(n^{3}\right)$ time.

\section{The Sum of Completion Times with the Investment Cost Minimization Problem}

In this section we study the problem $1 \mid G T, s_{i[r]}^{A}=$ $s_{i} r^{a \log _{2}(1-x) s}, A I L E, n_{i}=\bar{n} \mid \beta_{1} \sum C_{i j}+\beta_{2} k(x)$. We assume that each group has the same number of jobs. Our objective is to find the optimal group sequence, the job sequence, and the percentage reduction of the standard learning rate $x$.

For the problem $1 \mid G T, s_{i[r]}^{A}=s_{i} r^{a \log _{2}(1-x) s}, A I L E, n_{i}=$ $\bar{n} \mid \beta_{1} \sum C_{i j}+\beta_{2} k(x)$, by calculation, we present the following expression:

$$
\begin{aligned}
& \beta_{1} \sum C_{i j}+\beta_{2} k(x)=\sum_{i=1}^{m} \beta_{1}(n-(i-1) \bar{n}) s_{[i]} i^{a \log _{2}(1-x) s} \\
& +\sum_{i=1}^{m} \sum_{j=1}^{\bar{n}} \beta_{1}(n-(i-1) \bar{n}-j+1) \\
& \cdot p_{[i][j]} i^{a \log _{2}(1-x) s} j^{b \log _{2}(1-x) s}+\beta_{2} k(x) .
\end{aligned}
$$


Let $f_{2}(x)=\sum_{i=1}^{m} \beta_{1}(n-(i-1) \bar{n}) s_{[i]} i^{a \log _{2}(1-x) s}+\sum_{i=1}^{m} \sum_{j=1}^{\bar{n}} \beta_{1}(n-$ $(i-1) \bar{n}-j+1) p_{[i][j]} i^{i \log _{2}(1-x) s} j^{b \log _{2}(1-x) s}$ and $F_{2}(x)=f_{2}(x)+$ $\beta_{2} k(x)$.

From Lemma 3, we see that $f_{2}(x)$ is a convex function over the interval $\left[0, x_{\max }\right]$. Since $k(x)$ is a convex function, $F_{2}(x)$ is also a convex function over the interval $\left[0, x_{\max }\right]$. Hence, for a certain $x \in\left[0, x_{\text {max }}\right]$, the optimal sequence does not change. From the above expression, the group sequence does not affect the job sequence in each group.

Let $G_{2}(j)=\beta_{1}(n-(i-1) \bar{n}-j+1) j^{b \log _{2}(1-x) s}$. From Lemma 1 , we see that $G_{2}(j)$ is a nonincreasing function about $j$. From Lemma 4, for a given group sequence, the optimal internal job sequence can be obtained by assigning $p_{i[j]}$ to the corresponding $G_{2}(j)$ following a well-known result on two vectors given by Hardy et al. [20]. Since $G_{2}(j)$ is a nonincreasing function about $j, p_{i[j]}$ should be a nondecreasing sequence. Therefore, the optimal schedule is obtained by sequencing the jobs in each group in nondecreasing order of $p_{i[j]}$, i.e., $p_{i[1]} \leq p_{i[2]} \leq, \ldots, \leq p_{i\left[n_{i}\right]}, i=1,2,3, \ldots, m$ (the SPT rule). For a certain $x \in\left[0, x_{\max }\right]$, if the internal job sequence in each group is determined, the total cost depends on the group sequence. Therefore, we can determine the group sequence by solving the following assignment problem:

$$
\begin{aligned}
\text { Minimize } & \mathrm{Z}=\sum_{i=1}^{m} \sum_{r=1}^{m} \varphi_{i r} y_{i r} \\
\text { s.t. } \quad & \sum_{i=1}^{m} y_{i r}=1 \quad r=1,2, \ldots, m, \\
& \sum_{r=1}^{m} y_{i r}=1 \quad i=1,2, \ldots, m, \\
y_{i r}=0 & \text { or } 1 \\
& i=1,2, \ldots, m, r=1,2, \ldots, m .
\end{aligned}
$$

where $\varphi_{i r}=\beta_{1}(n-(r-1) \bar{n}) s_{i} r^{a \log _{2}(1-x) s}+\sum_{j=1}^{\bar{n}} \beta_{1}(n-(r-$ $1) \bar{n}-j+1) p_{i[j]} r^{a \log _{2}(1-x) s} j^{b \log _{2}(1-x) s}$.

From the above expression, the optimal $x$ depends on the sign of the first derivative of $F_{2}(x)$, i.e., $F_{2}^{\prime}(x)$. If $F_{2}^{\prime}(x)$ is positive, we should allocate the minimal value to $x$, i.e., $x=0$. If $F_{2}^{\prime}(x)$ is negative, we should allocate the maximal value to $x$, i.e., $x=x_{\text {max }}$. If $F_{2}^{\prime}(x)$ is equal to 0 , any value between 0 and $x_{\text {max }}$ will not affect the total cost.

Based on the above analysis, we propose the following algorithm to solve this problem.

\section{Algorithm 7.}

Step 1. Arrange the jobs in each group in the SPT order, i.e.,

$$
p_{i[1]} \leq p_{i[2]} \leq, \ldots, \leq p_{i\left[n_{i}\right]}, \quad i=1,2, \ldots, m .
$$

Step 2. Give a value to $x$, which $x \in\left[0, x_{\max }\right]$, schedule the groups by $\left(A P_{2}\right)$.

Step 3. Take the first derivative of $F_{2}(x)$
Set

$$
x^{*}= \begin{cases}0, & \text { if } F_{2}^{\prime}(x)>0, \\ x_{\text {max }}, & \text { if } F_{2}^{\prime}(x)<0, \\ x \in\left[0, x_{\text {max }}\right], & \text { if } F_{2}^{\prime}(x)=0 .\end{cases}
$$

Step 4. Output the optimal job sequence, optimal group sequence, and optimal percentage reduction of the standard learning rate $x^{*}$. Similar to the above analysis, the complexity of Algorithm 7 is at most $O\left(n^{3}\right)$. Thus, we obtain the following result.

Theorem 8. The $1 \mid G T, s_{i[r]}^{A}=s_{i} r^{a \log _{2}(1-x) s}, A I L E, n_{i}=$ $\bar{n} \mid \beta_{1} \sum C_{i j}+\beta_{2} k(x)$ problem is solvable in $O\left(n^{3}\right)$ time.

\section{Conclusions}

In this paper we consider single-machine group scheduling with autonomous and induced learning. The objectives are to minimize a linear combination the makespan and the investment cost and a linear combination the total completion time and the investment cost. We first derive that the problems to minimize the weighted sum of the makespan and the investment cost are polynomially solvable. Then we prove that the problems to minimize the weighted sum of the total completion time and the investment cost are polynomially solvable under certain condition. In future research it is worth considering more general learning models in other machine environments or extend our model with other objective functions.

\section{Appendix}

See Lemma 1.

Proof. For $G(r)=\delta(r) r^{m \log _{2}(1-x) s}$, taking the first derivatives of $G(r)$ with respect to $r$, we get

$$
\begin{aligned}
& G^{\prime}(r) \\
& \quad=\left(\delta^{\prime}(r) r+\delta(r) m \log _{2}(1-x) s\right) r^{-1+m \log _{2}(1-x) s} \\
& \quad<0,
\end{aligned}
$$

on $m \geq 1,0 \leq x \leq x_{\max }<1, r \geq 1, \delta(r) \geq 1, \delta(r)$ is a nonincreasing function. Hence, $G(r)$ is a nonincreasing function in $r$.

\section{See Lemma 2.}

Proof. For $h(x)=\delta(r) r^{m \log _{2}(1-x) s}$, taking the second derivatives of $h(x)$ with respect to $x$, we get

$$
\begin{aligned}
h^{\prime}(x) & =\delta(r) r^{m \log _{2}(1-x) s} \frac{m \log _{2} r}{(x-1)}, \\
h^{\prime \prime}(x) & =\delta(r) r^{m \log _{2}(1-x) s} \frac{m \log _{2} r\left(m \log _{2} r-1\right)}{(x-1)^{2}} .
\end{aligned}
$$

Since $m \geq 1, h^{\prime \prime}(x)=0$ when $r=1$ and $h^{\prime \prime}(x) \geq 0$ if $r>1$. Therefore, $h(x)$ is a convex function over $\left[0, x_{\text {max }}\right]$. 


\section{See Lemma 3.}

Proof. For $\rho(x)=\delta_{1}(i, j) i^{a \log _{2}(1-x) s}+\delta_{2}(i, j) i^{a \log _{2}(1-x) s} j^{b \log _{2}(1-x) s}$, taking the second derivatives of $\rho(x)$ with respect to $x$, we get

$$
\begin{aligned}
& \rho^{\prime}(x)=\delta_{1}(i, j) i^{a \log _{2}(1-x) s} \frac{a \log _{2} i}{x-1}+\delta_{2}(i, j) \\
& \cdot i^{a \log _{2}(1-x) s} j^{b \log _{2}(1-x) s} \frac{a \log _{2} i+b \log _{2} j}{x-1} \\
& \rho^{\prime \prime}(x)=\delta_{1}(i, j) i^{a \log _{2}(1-x) s} \frac{a \log _{2} i\left(a \log _{2} i-1\right)}{(x-1)^{2}} \\
& +\delta_{2}(i, j) i^{a \log _{2}(1-x) s} j^{b \log _{2}(1-x) s} \\
& \cdot \frac{\left(a \log _{2} i+b \log _{2} j\right)\left(a \log _{2} i+b \log _{2} j-1\right)}{(x-1)^{2}} .
\end{aligned}
$$

Since $a \geq 1, b \geq 1, \rho^{\prime \prime}(x)=0$ when $i=1, j=1$ and $\rho^{\prime \prime}(x) \geq$ 0 if $i>1, j>1$. Therefore, $\rho(x)$ is a convex function over $\left[0, x_{\text {max }}\right]$.

\section{Data Availability}

The data used to support the findings of this study are included within the article.

\section{Conflicts of Interest}

The authors declare that they have no conflicts of interest.

\section{Acknowledgments}

This work is partly supported by the National Natural Science Foundation of China (71532015).

\section{References}

[1] D. Biskup, "Single-machine scheduling with learning considerations," European Journal of Operational Research, vol. 115, no. 1, pp. 173-178, 1999.

[2] T. P. Wright, "Factors affecting the cost of airplanes," Journal of the Aeronautical Sciences, vol. 3, no. 4, pp. 122-128, 1936.

[3] T. C. E. Cheng and G. Wang, "Single machine scheduling with learning effect considerations," Annals of Operations Research, vol. 98, pp. 273-290, 2000.

[4] J. Qian and G. Steiner, "Fast algorithms for scheduling with learning effects and time-dependent processing times on a single machine," European Journal of Operational Research, vol. 225, no. 3, pp. 547-551, 2013.

[5] J.-B. Wang and J.-J. Wang, "Flowshop scheduling with a general exponential learning effect," Computers \& Operations Research, vol. 43, pp. 292-308, 2014.

[6] R. a. Rudek, "On single processor scheduling problems with learning dependent on the number of processed jobs," Applied Mathematical Modelling: Simulation and Computation for Engineering and Environmental Systems, vol. 37, no. 3, pp. 1523-1536, 2013.
[7] M. Liu, "Parallel-machine scheduling with past-sequencedependent delivery times and learning effect," Applied Mathematical Modelling, vol. 37, no. 23, pp. 9630-9633, 2013.

[8] L. Shen and Y.-B. Wu, "Single machine past-sequencedependent delivery times scheduling with general positiondependent and time-dependent learning effects," Applied Mathematical Modelling, vol. 37, no. 7, pp. 5444-5451, 2013.

[9] C.-C. Wu, W.-C. Lee, and M.-J. Liou, "Single-machine scheduling with two competing agents and learning consideration," Information Sciences, vol. 251, pp. 136-149, 2013.

[10] G. Li, X.-Y. Wang, J.-B. Wang, and L.-Y. Sun, "Worst case analysis of flow shop scheduling problems with a time-dependent learning effect," International Journal of Production Economics, vol. 142, no. 1, pp. 98-104, 2013.

[11] R. a. Rudek, "Scheduling on parallel processors with varying processing times," Computers \& Operations Research, vol. 81, pp. 90-101, 2017.

[12] X. Zhang, L. Sun, and J. Wang, "Single machine scheduling with autonomous learning and induced learning," Computers \& Industrial Engineering, vol. 66, no. 4, pp. 918-924, 2013.

[13] D. Biskup and D. Simons, "Common due date scheduling with autonomous and induced learning," European Journal of Operational Research, vol. 159, no. 3, pp. 606-616, 2004

[14] C.-M. Wei and J.-B. Wang, "Single machine quadratic penalty function scheduling with deteriorating jobs and group technology," Applied Mathematical Modelling: Simulation and Computation for Engineering and Environmental Systems, vol. 34, no. 11, pp. 3642-3647, 2010.

[15] S.-J. Yang, "Group scheduling problems with simultaneous considerations of learning and deterioration effects on a singlemachine," Applied Mathematical Modelling, vol. 35, no. 8, pp. 4008-4016, 2011

[16] W.-C. Lee and C.-C. Wu, "A note on optimal policies for two group scheduling problems with deteriorating setup and processing times," Computers \& Industrial Engineering, vol. 58, no. 4, pp. 646-650, 2010.

[17] J. Bai, Z.-R. Li, and X. Huang, "Single-machine group scheduling with general deterioration and learning effects," Applied Mathematical Modelling, vol. 36, no. 3, pp. 1267-1274, 2012.

[18] W. C. Lee and Z. S. Lu, "Group scheduling with deteriorating jobs to minimize the total weighted number of late jobs," Applied Mathematics and Computation, vol. 218, no. 17, pp. 8750-8757, 2012.

[19] W.-C. Lee and C.-C. Wu, "A note on single-machine group scheduling problems with position-based learning effect," Applied Mathematical Modelling, vol. 33, no. 4, pp. 2159-2163, 2009.

[20] G. H. Hardy, J. E. Littlewood, and G. Polya, Inequalities, Cambridge University Press, London, UK, 1967. 


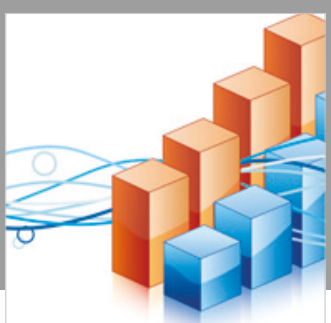

Advances in

Operations Research

\section{-n-m}
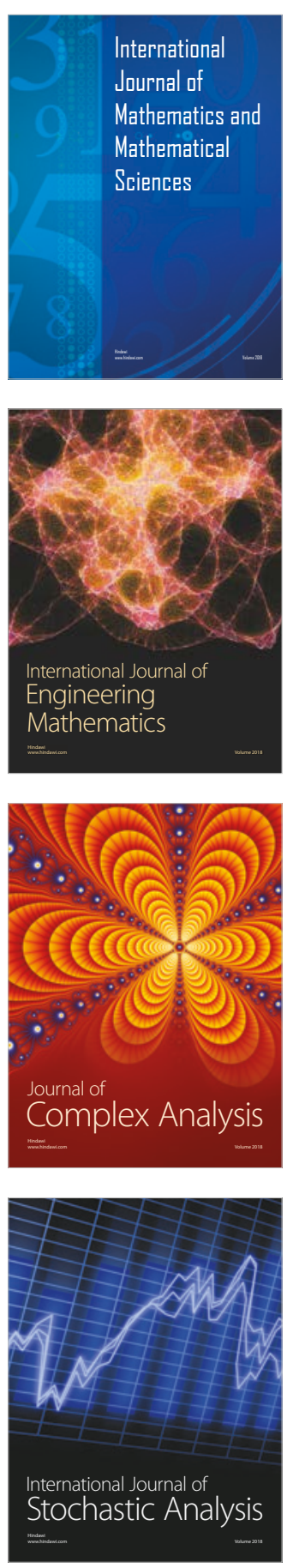
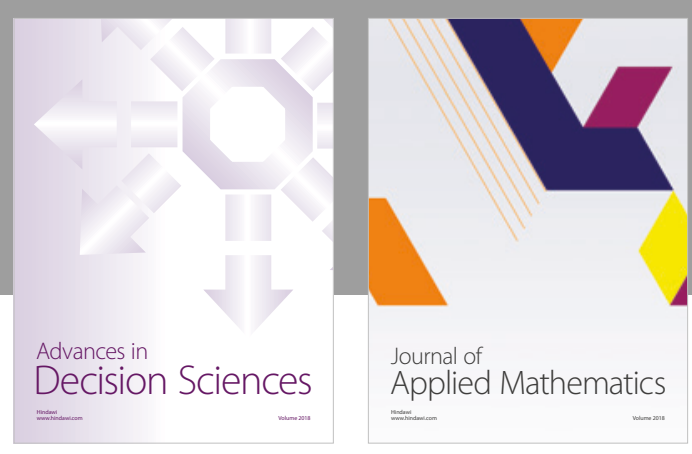

Journal of

Applied Mathematics
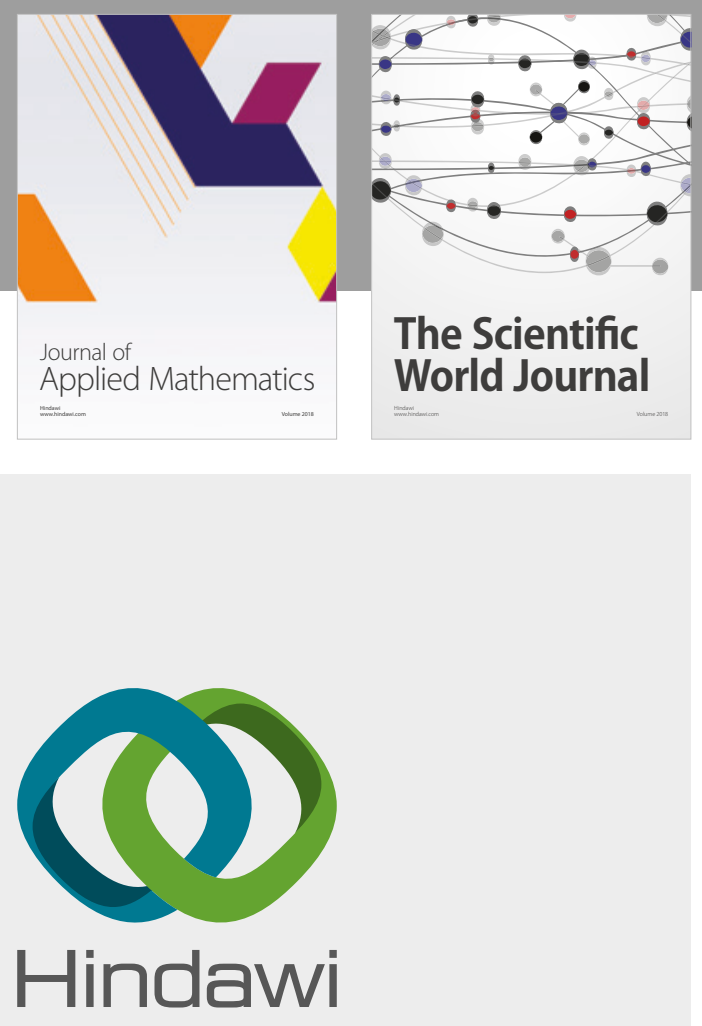

Submit your manuscripts at

www.hindawi.com

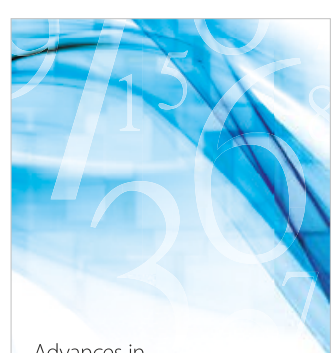

Advances in
Numerical Analysis
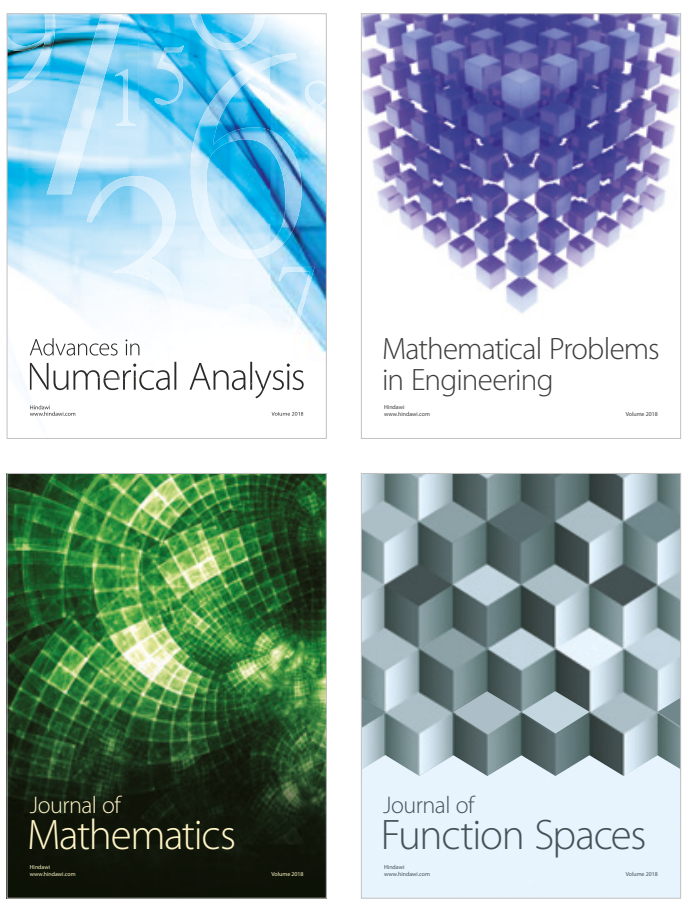

Mathematical Problems in Engineering

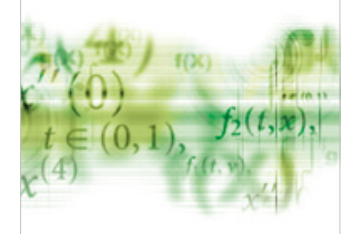

International Journal of

Differential Equations

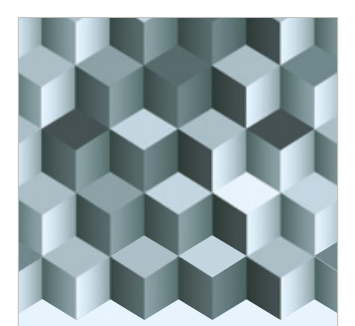

Journal of

Function Spaces

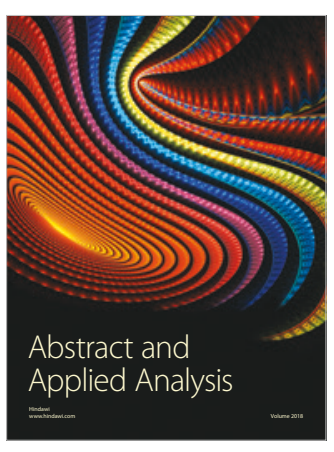

The Scientific

World Journal

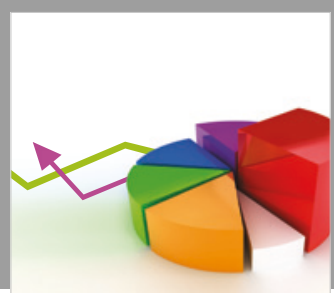

Journal of

Probability and Statistics
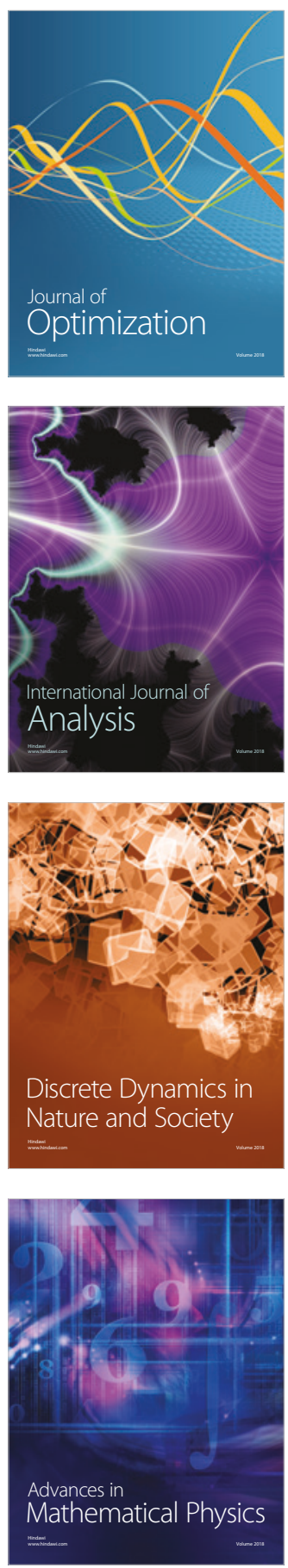